\title{
Advanced THz MIMO Sparse Imaging Scheme Using Multi-pass Synthetic Aperture Focusing and Low Rank Matrix Completion Techniques
}

\author{
Shaoqing Hu Member, IEEE, Chao Shu, Yasir Alfadhl, Senior Member, IEEE, and Xiaodong Chen, Fellow, IEEE
}

\begin{abstract}
This paper proposes an advanced THz multipleinput-multiple-output (MIMO) near-field sparse imaging scheme for target detection that uses single-pass synthetic aperture focusing and multi-pass interferometric synthetic aperture focusing techniques to improve imaging effectiveness and efficiency. It benefits from the MIMO of linear sparse periodic array (SPA) and random sparse imaging to reduce sampling data as well as system cost. Both simulated and proof-of-concept experimental results have verified the scheme, revealing that the single-pass synthetic aperture imaging approach is sufficient to identify pure metallic targets with $3.87 \%$ of $\lambda / 2$ sampling data. And multi-pass interferometric synthetic aperture imaging approach is capable of improving image quality on image signal to noise ratio and contrast, which is ideal for detecting more challenging targets. The random sparse imaging with help of low rank matrix completion (LRMC) technique has shown promising potential of achieving equivalent image quality when further reducing 43.75\% data in the experiment of 5-pass imaging on a complex target, this corresponds to $82.51 \%$ data reduction compared to $\lambda / 2$ sampling.
\end{abstract}

Index Terms-Interferometric synthetic aperture radar (InSAR), low rank matrix completion (LRMC), multiple-inputmultiple-output (MIMO), personnel screening, security detection, sparse periodic array (SPA), under-sampled sparse imaging.

\section{INTRODUCTION}

$\mathbf{M}$ ICROWAVE and sub-millimeter wave imaging for target detection has been developed in last two decades, it faces low spatial resolution due to low operation frequency [1]-[6]. THz wave, referring to the frequency band between $100 \mathrm{GHz}$ and $10 \mathrm{THz}$, has a short wavelength, non-ionizing characteristic and strong penetrating ability which is promising to applications for target detection or personnel screening [7]-[9]. This kind of imaging systems whatever frequency band is used can be categorized into passive, active imaging systems and their combination. The passive imaging system utilizes emissivity or temperature differences of objects to form the target images [10]-[15]. It is capable of achieving a fast imaging rate due to exempting from transmitting signal but challenging in achieving a sufficient sensitivity to detect a small noise equivalent temperature difference (NETD or $\mathrm{NE} \Delta \mathrm{T}$ ) below $1 \mathrm{~K}$, which is desirable to detect items warmed up by body contact. On the contrary, the active imaging system

Shaoqing Hu is with Department of Electronic and Electrical Engineering, College of Engineering, Design \& Physical Sciences, Brunel University London, Greater London, UB8 3PH, UK (e-mail: shaoqing.hu@ brunel.ac.uk).

Chao Shu, Yasir Alfadhl and Xiaodong Chen are with School of Electronic Science and Computer Science, Queen Mary University of London, London, E1 4NS, UK. is capable of achieving a superior imaging performance because of power source illumination and collecting the stronger reflections from target. The fast electronic scanning of a linear array integrated with mechanical scanning of array movement provides a possible solution to balancing the high frame rate and low system cost. The state-of-the-art MIMO array are used for electronic scanning [16]-[20] including multi-static linear sparse periodic array (SPA) [21]-[24], which has an advantage in reducing the array elements but maintaining high image quality. However, most MIMO imaging systems use sampling spacing on the order of $\lambda / 2$ by Nyquist sampling criterion to avoid aliasing [22]. This will cause the increase of data acquisition time and system cost due to a large number of sampling points and channels at high frequency band. For example, there are 384 antenna elements in each of transmitter and receiver linear array imaging system working at 24.25$30 \mathrm{GHz}$ [18] while a total of $736 \mathrm{Tx}$ antennas and $736 \mathrm{Rx}$ antennas are used in a planar imaging system working at 72$80 \mathrm{GHz}$ [19]. We have investigated the feasibility of large sampling spacing in SPA for $\mathrm{THz}$ imaging. The maximum Tx or Rx element spacing is preferred to be determined by the interested field of view without incurring ghost images [25]. Large sampling spacing is applicable but it will cause image quality of signal to noise ratio (SNR) and contrast deteriorate, which is ideal for detecting pure metallic targets as demonstrated in this paper. This imaging approach is termed single-pass synthetic aperture focusing imaging. In addition, we investigated multi-pass interferometric synthetic aperture focusing technique to improve image quality [26]. Interferometric technique has been widely used in airborne synthetic aperture radar (SAR) and inverse synthetic aperture radar (ISAR) to form a 3D image [27]-[32]. It uses phase correlation resulting from either multiple receivers (usually 3 ) or different passes (usually 2) to gain the third dimensional information compared to traditional 2D SAR/ISAR image. It utilizes mono-static transceivers compared to the imaging distance with single or a few receivers [30]-[32]. However, we firstly apply multi-pass interferometric technique into near field $\mathrm{THz}$ sparse imaging system with multi-static MIMO array for potential application of security/personnel screening. It improves imaging performance without deploying more channels so as to maintain low system cost. We propose two detection working simultaneously called dual-mode operation that allows to cease the detection earlier, this will increase the detection efficiency compared to directly using $\lambda / 2$ Nyquist sampling in one pass. 
This article has been accepted for publication in a future issue of this journal, but has not been fully edited. Content may change prior to final publication. Citation information: DOI: 10.1109/TMTT.2021...

IEEE TRANSACTIONS ON MICROWAVE THEORY AND TECHNIQUES, VOL. XX, NO. XX, XXXX 2021

The other significant novelty of proposed work is integrating random sparse imaging into the multi-pass synthetic aperture focusing technique to reduce the sampling data, time and associated system cost. Sparse imaging with a linear sparse array and specific algorithm has been proposed [20]. However, the center frequency in operation is low as $35 \mathrm{GHz}$. In addition, the targets are three simplified metallic cylinders at a short distance of $0.5 \mathrm{~m}$ and the proposed algorithm would suffer from the heavy computational complexity for large or complex scenario. Another two most popular reconstruction algorithms for random sparse imaging are compressive sensing (CS) and low rank matrix completion (LRMC) [33], [34]. However, the performance of CS-based method depends on the design of measurement matrix [35] and it is sensitive to noise [36]. Besides, it will cost much PC resource and time to reconstruct images due to sparse representation (a round transformations between two domains). By contrast, LRMC technique is an alternative but superior technique to reconstruct the missing samples exempting from the sparse representation [37], [38]. With the advantages of high reconstruction accuracy, LRMC has gained interests in various applications, such as radar changing imaging [36], remote SAR imaging [39], [40] and the latest W-band 0.4m-SAR imaging for target detection [34]. However, there is very little discussion about $\mathrm{THz}$ sparse imaging with LRMC technique and multi-pass synthetic aperture focusing technique for target detection, far target distances in particular. Thus it has been proposed and investigated in this paper, achieving successful imaging at 220 $\mathrm{GHz}$ for detecting finer, more challenging targets at a far distance of $1.4 \mathrm{~m}(<54.10 \mathrm{~m}$ of far field distance) in both simulation and proof-of-concept experiments. The results have demonstrated its capability of reducing sampling points as well as array elements but maintaining a high-quality image at $\mathrm{THz}$ band, which is particularly vital in reducing system cost of planar imaging systems.

In addition, another contribution of this paper is that a systematic approach of computational imaging simulation has been proposed that involves the theoretical analysis in MATLAB and computational electromagnetic imaging simulation with $\mathrm{FEKO}^{\mathrm{TM}}$. Computational imaging simulation is challenging due to a long distance of free space involved, especially at $\mathrm{THz}$ band of tiny mesh size. Thus, targets are usually simplified models like simple points, small scatters or metallic plates [2], [17], [34], [41], [42]. The proposed approach is capable of modelling various imaging scenarios.

The rest of paper is organized as follows. Section II introduces the fundamental theoretical analysis and computational electromagnetic (EM) simulation of an imaging scheme. Section III introduces the LRMC technique and corresponding simplified single value thresholding (SVT) algorithm for the proposed sparse random imaging. Section IV uses simulation examples to show the effectiveness of the proposed computational imaging simulation and LRMC-SVT-based image reconstruction. Section V gives proof-of-concept experiments and measured imaging results including single-pass synthetic aperture imaging and multi-pass interferometric synthetic aperture imaging of two kinds of targets: a pure metallic target and a complex target with dielectric substrate. Section VI and VII give discussions and conclusions.

\section{Systematic Computational Simulation ON SYNTHETIC APERTURE IMAGING}

Theoretical analysis using self-programmed codes leads to initial imaging system design while modeling and simulation using computational electromagnetic (CEM) software provides an effective way to optimize and validate the imaging performance prior to practical implementation. Therefore, a complete systematic simulation study approach including preliminary theoretical analysis in MATLAB and CEM imaging in $\mathrm{FEKO}^{\mathrm{TM}}$ has been proposed.

\section{A. Theoretical Analysis in MATLAB}

The MIMO array used also known as SPA consists of $N t$ transmitter (Tx) elements with a spacing of dt, half placed at two ends of the $N r$ receiver ( $\mathrm{Rx}$ ) elements with a spacing of $\mathrm{dr}$ that equals to $0.5 \cdot N t \cdot d t$, as shown in Fig.1 [25]. The electronic scanning along one direction such as the horizontal direction shown in Fig. 2 is achieved by sequentially switched on and off transmitter elements, when each transmitter is on, all receiver elements are used to record the echo data. The sequence number of SPA and corresponding Tx-Rx pairs for recorded data in each row is shown in Table I. Afterwards, the SPA moves to next position along the other direction, which is called mechanical scanning, and previous recording is repeated. This process is repeated until full aperture data are sampled [25]. This one (the number $N=1$ ) planar scanning leads to single(one)-pass synthetic aperture imaging. In order to improve imaging performance, an additional degree of freedom of SPA position along the electronic scanning direction with a step of $d s$ is introduced. It can be either constant for simplicity or variable to achieve flexible sampling configuration. The total number is $N(>1)$, donating $N$ pass. These muti-pass datesets are phase correlated so they can be mathematically focused with multi-pass interfermetric synthetic focusing technique.

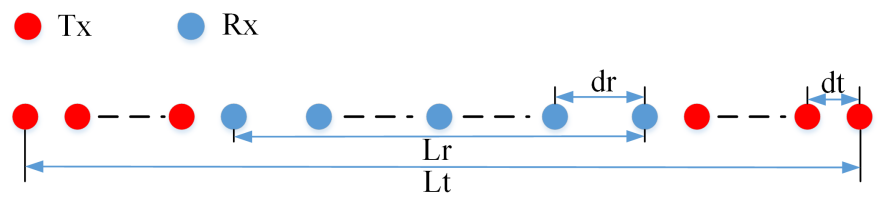

Fig. 1. Linear Sparse Periodic Array.

Therefore, we can gain a planar PSF of this SPA imaging system-the product of planar PSFs of transmitter and receiver

TABLE I

SPA TR PAIRS ON ELECTRONIC SCANNING DIRECTION WHICH IS REPEATED WITH MECHANICAL SCANNING ON OTHER DIRECTION.

\begin{tabular}{ccc}
\hline Sequence & $\mathrm{Tx}^{1} \cdots \mathrm{Tx}^{\mathrm{Nt} / 2}, \mathrm{Rx}^{1} \cdots \mathrm{Rx}^{\mathrm{Nr}}, \mathrm{Tx}^{\mathrm{Nt} / 2+1} \cdots \mathrm{Tx}^{\mathrm{Nt}}$ \\
1st half & $\mathrm{Rx}^{1} \mathrm{Tx}^{1} \sim \mathrm{Rx}^{1} \mathrm{Tx}^{\mathrm{Nt} / 2} \cdots \mathrm{Rx}^{\mathrm{Nr}} \mathrm{Tx}^{1} \sim \mathrm{Rx}^{\mathrm{Nr}} \mathrm{Tx}^{\mathrm{Nt} / 2}$ \\
2nd half & $\mathrm{Rx}^{1} \mathrm{Tx}^{\mathrm{Nt} / 2+1} \sim \mathrm{Rx}^{1} \mathrm{Tx}^{\mathrm{Nt}} \cdots \mathrm{Rx}^{\mathrm{Nr}} \mathrm{Tx}^{\mathrm{Nt} / 2+1} \sim \mathrm{Rx}^{\mathrm{Nr}} \mathrm{Tx}^{\mathrm{Nt}}$ \\
\hline
\end{tabular}




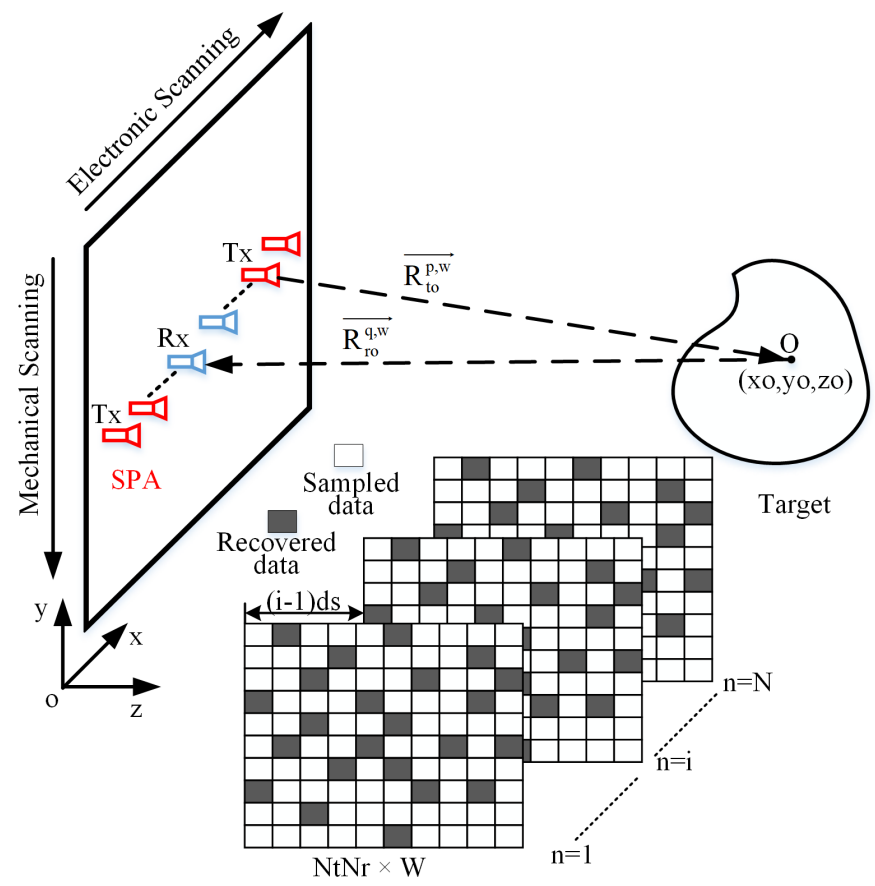

Fig. 2. SPA Planar Scanning Scheme in Simulation.

arrays, using Generalized Synthetic Aperture Focusing Technique (GSAFT) in

$$
P S F(S P A)=\sum_{n=1}^{N} \sum_{w=1}^{W} \sum_{p=1}^{N t} \sum_{q=1}^{N r} e^{j k\left(\left|\vec{R}_{t o, n}^{p, w}\right|+\left|\vec{R}_{r, n}^{q, w}\right|\right)} .
$$

which is also the imaging system response. The $\vec{R}_{t o, n}^{p, w}$ and $\vec{R}_{r, n}^{q, w}$ are the vectors in the $n$th pass between the position of $p$ th transmitting antenna at $w$ th mechanical scanning position and the position of $q$ th receiving antenna at $w$ th mechanical scanning position to the target point $o$.

Equation (1) depict the 2D point spread functions whose slice cuts are the corresponding 1D point spread functions. PSFs provide a method to assess the imaging performance of cross range spatial resolution and artifacts. For example, Fig. 3 shows the perspective view and top view of a PSF derived from (1). The first null implies the cross range spatial resolution while the side lobe level predicts how likely the artifacts arise.

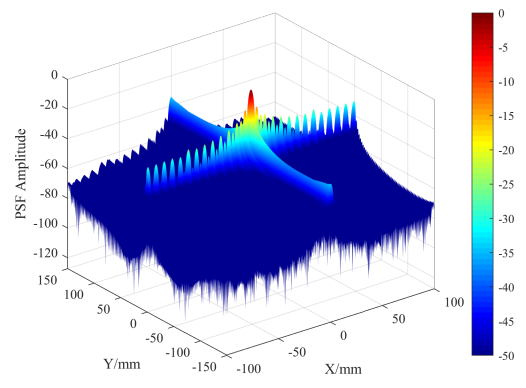

(a)

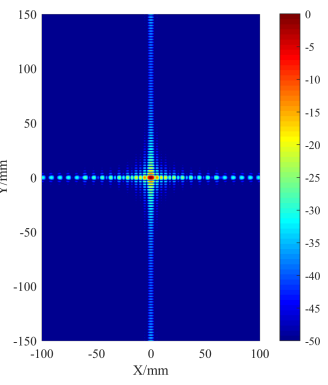

(b)
Fig. 3. 2D PSF (a) Perspective View (b) Top View.
In addition, the theoretical cross range resolution for an imaging or radar system can be predicted by

$$
\left.\delta_{\text {cross-range }} \approx \frac{\lambda_{c}}{4 \cdot \sin (\theta / 2)}\right|_{\theta=\min \left(\theta_{H P B W}, 2 \arctan \left(\frac{L}{2 D}\right)\right)},
$$

where $\lambda_{c}$ is wavelength of center frequency, $L$ is the scanning length along either direction, $\theta$ is the minimum angle between Half Power Beam Width (HPBW) of receiver antenna and subtended angle by scanning aperture. For an aperture-limited imaging system in which target distance $D$ is much larger than scanning aperture length or the receiver antenna has about $180^{\circ}$ beam width, equation (2) can be approximated by

$$
\delta_{\text {limited-aperture }} \approx \frac{\lambda_{c}}{2 \cdot L} \cdot D,
$$

where $2 \cdot L$ replaced by the effective aperture length of $L d=$ $L t+L r$ is widely used in SPA electronic scanning [25].

The theoretical range resolution can be predicted by

$$
\delta_{\text {range }}=\frac{c}{2 B},
$$

where $c$ is light speed constant and $B$ is operation bandwidth of imaging system.

Equation $(1) \sim(4)$ give the theoretical analysis of resolutions and contrast (side lobe level) on an imaging system in MATLAB. Following on the design guideline we proposed in [25], it leads to the configuration of SPA and scanning intervals according to the desired imaging performance. The design will then be verified by computational electromagnetic simulation in $\mathrm{FEKO}^{\mathrm{TM}}$.

\section{B. Computational EM Imaging Simulation in FEKO ${ }^{T M}$}

After the echo datasets (or S parameters) are collected, the corresponding multi-pass interferometric synthetic aperture focusing image can be reconstructed by multiplying $\mathrm{S}$ parameters and system response (PSF in (1)) as

$$
\begin{aligned}
g\left(r_{o}\right)= & \sum_{n=1}^{N} \sum_{w=1}^{W} \\
& \sum_{p=1}^{N t} \sum_{q=1}^{N r} S\left(\vec{R}_{t o, n}^{p, w}, \vec{R}_{r o, n}^{q, w}\right) \cdot e^{j k\left(\left|\vec{R}_{t o, n}^{p, w}\right|+\left|\vec{R}_{r o, n}^{q, w}\right|\right)} .
\end{aligned}
$$

If $N=1$, it is simplified as single-pass synthetic aperture focusing image. In addition to resolutions, image signal to noise ratio (SNR) defined by

$$
S N R_{\text {image }}=\frac{\overline{B_{\text {bright }}}-\overline{B_{\text {dark }}}}{\sigma\left(B_{\text {dark }}\right)},
$$

can be used to quantitatively assess the image contrast (quality), where $\overline{B_{\text {bright }}}$ and $\overline{B_{\text {dark }}}$ are the mean brightness of the target area and non-target (dark) area. $\sigma\left(B_{\text {dark }}\right)$ is the standard deviation of the non-target (dark) area brightness [43].

$S$ parameters can be gained by computational electromagnetic (CEM) simulation that provides an effective way to evaluate imaging system prior to the practical implementation. Full wave solutions in CEM simulation provide reliable results but require fine mesh size and more PC resources. One of full wave solutions-multilevel fast multipole method (MLFMM) 
in $\mathrm{FEKO}^{\mathrm{TM}}$ is much more efficient to reduce the memory complexity and solving complexity. Asymptotic solutions in CEM simulation including physical optics (PO) and raylaunching geometrical optics (RL-GO) provide reasonably accurate results by solving Maxwell Equations with appropriate assumptions and approximations. They save much PC resources and computational time when applied to electrically large targets [44], [45]. Therefore, $\mathrm{FEKO}^{\mathrm{TM}}$ is suitable for the imaging simulation that involves a long free space of target distance and large, complex targets. The featured hybrid solutions in $\mathrm{FEKO}^{\mathrm{TM}}$ that combines full wave and asymptotic solutions can facilitate simulation on challenging scenarios with less computational resources but providing required accuracy at the same time. For the simplest imaging scenario that only involves a small array and a pure small metallic target, it is suitable to use either the default method of moments (MOM) or hybrid solution of MLFMM/MOM. For the intermediate imaging scenario that involves a larger array, long target distance or large pure metallic target, the MOM/PO or MLFMM/PO hybrid solution can be adopted in which the MOM or MLFMM are used as the global solver while the PO is used as the local solver for the target. Besides, the large element physical optics (LE-PO) can be used instead of PO, which allows for much larger mesh size to save PC randomaccess memory and computational time. However, if the target includes the dielectric, the PO-based hybrid simulation method is not applicable. Thus, the RL-GO method can be applied in this imaging scenario. Geometrical optics (GO) leads to the mesh storage reduced remarkably, so it is very suitable for electrically large targets of perfect electric conductor and dielectric objects with multiple reflections. Taking mesh element size of triangular element as an example, the side length in MOM, MLFMM and PO should be shorter than approximately $\frac{\lambda}{5 \cdots 6}$ and an edge length of $\frac{\lambda}{8 \cdots 10}$ is preferred. The allowed edge length in LE-PO for when near field requests are present should be $2 \lambda$. If only far field requests are present, the triangles only need to represent the geometry accurately, regardless of frequency or wavelength. For dielectric surfaces to be solved using the GO method, the mesh size is independent of the solution frequency so the maximum triangular mesh element size should be chosen such that the geometry of the surface is well represented. It is worth mentioning that the RL-GO in hybrid simulation can only be coupled with the MOM rather than MLFMM. It is indicated that the computational cost including PC resources and simulation time will reduce with later approaches but accuracy reduces because of assumptions and approximations used. Therefore, a simulation approach that properly meets demands should be chosen. Beside, if the array elements are uniform, we can use the equivalent source of element's far field placed at the elements' positions instead of the geometric model of array to further significantly save the computational time, this method is effective to deal with imaging scenario deploying a large array and its interaction with large targets [46].

In summary, the systematic work flow of this computational electromagnetic imaging simulation study has been concluded in Fig.4. Based on the desired imaging specifications, the configurations of MIMO array (SPA) and mechanical scanning

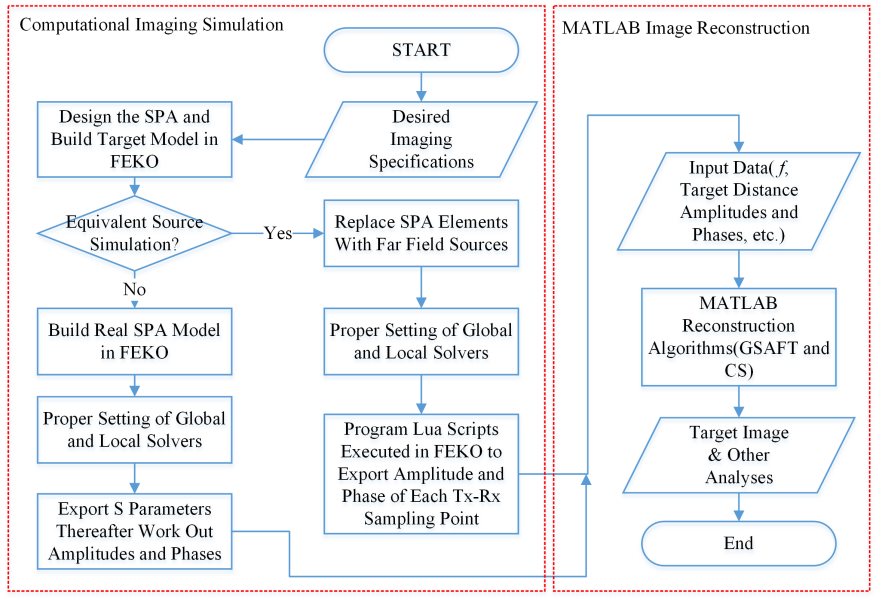

Fig. 4. Flow chart of computational electromagnetic imaging simulation study.

are firstly designed according to the analysis according to (1) $\sim$ (4) and design guideline we proposed in [25]. Then, the computational simulation is conducted in $\mathrm{FEKO}^{\mathrm{TM}}$ in which equivalent source simulation method might be adopted to save computational time and PC resources for complex imaging scenarios. However, the echo data (amplitudes and phases) need to be extracted by self-programmed lua scripts in PostFEKO if equivalent source simulation is used. Thereafter, the simulated data are input to reconstruct the target image based on algorithm like GSAFT in (5).

\section{LOW RANK MATRix COMPletion TECHNiQUe FOR UNDER-SAMPLED SPARSE IMAGING}

Due to multi-pass phase correlated datasets, the sampling time and data processing load both increase. We aim to explore random sparse sampling and reconstruction approach to alleviate these issues. It is vital in implementing the planar imaging system which will remove part of the TR channels. The full $\mathrm{S}$ parameters in (5) for reconstruction is $S_{f u l l}=\left\{S_{N t N r \times W}\right\}_{n=i}^{N}$. They can be either fully sampled or approximated with few observations-random under-sampled data $S_{\text {sparse }}$ denoted in black and corresponding completed data by LRMC denoted in white, as shown in Fig.5.

$$
S_{\text {sparse }}(i, j)=P \cdot S_{\text {full }}= \begin{cases}S_{\text {full }}(i, j), & (i, j) \text { sampled } \\ 0, & \text { otherwise }\end{cases}
$$

where $P$ is a sparse sampling matrix consisting of 0 (black) and 1 (white) to deploy different sampling rate and pattern for study. In practical product, only active samples and associated channels can be implemented. According to related theory [37], [47], the missing echo can be completed by solving the following nuclear norm minimization optimization problem:

$$
\begin{aligned}
& \operatorname{minimize}\left\|\hat{S}_{\text {full }}\right\|_{*} \\
& \text { subject to }\left\|\hat{S}_{\text {full }}-S_{\text {sparse }}\right\|_{F} \leq \delta
\end{aligned}
$$

where $\left\|\hat{S}_{\text {full }}\right\|_{*}$ is the nuclear norm of recovery matrix $\hat{S}_{\text {full }}$, which is the sum of its singular values [47], and $\|*\|_{F}$ refers to 
This article has been accepted for publication in a future issue of this journal, but has not been fully edited. Content may change prior to final publication. Citation information: DOI: 10.1109/TMTT.2021...

IEEE TRANSACTIONS ON MICROWAVE THEORY AND TECHNIQUES, VOL. XX, NO. XX, XXXX 2021

the Frobenius norm, which is the square root of the sum of the absolute squares of its elements. There are many algorithms to solve (8) such as Principal Component Pursuit by Alternating Directions and Single Value Thresholding (SVT) [37], [38], [48], which is not the focus of this paper. Thus we do not address details and have proposed to simplify the traditional SVT algorithm by removing specifying the number of singular values in computation [37], [47], as introduced in Table II

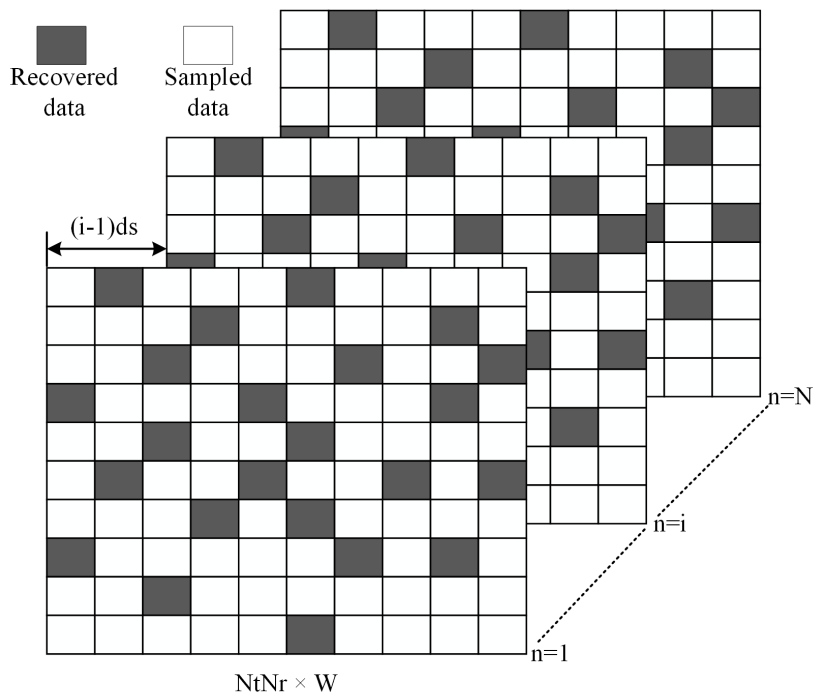

Fig. 5. Illustration of $\hat{S}_{f u l l}$ data.

TABLE II

SiMPLIFIED SVT LRMC SPARSE IMAGING ALGORITHM.

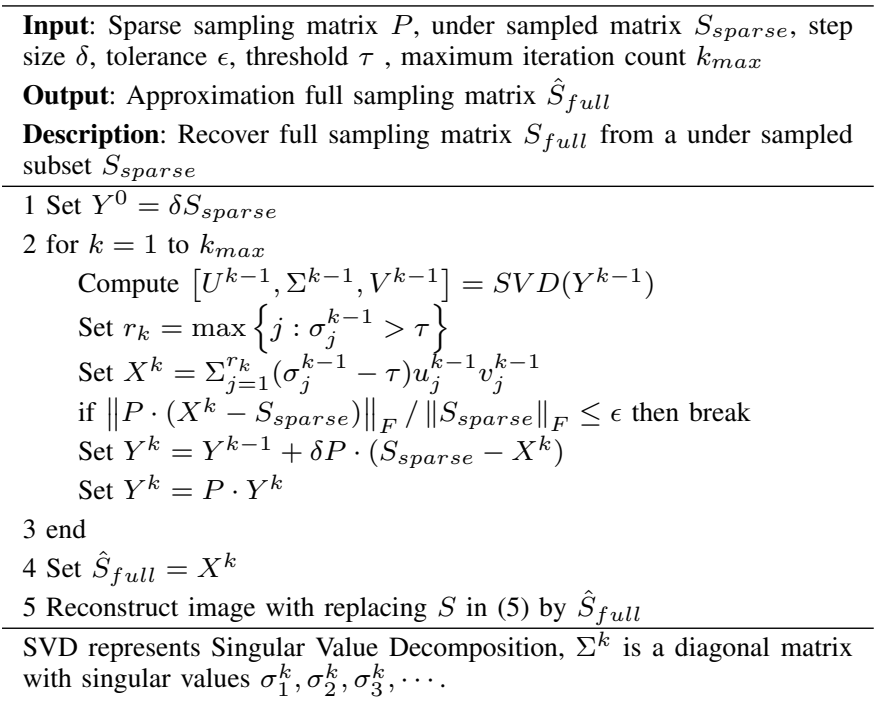

\section{Computational Imaging Simulation And RESULTS}

Using above proposed method, we carried out computational imaging simulation study prior to the practical implementation. For example, there are two configurations listed in Table III. The corresponding reconstructed single-pass synthetic aperture focusing images are shown in Fig.6 and image
TABLE III

Simulation CONFIgURATIONS $(8 \mathrm{TX} \& 8 \mathrm{RX}$ WITH TX ELEMENT SPACING $d t=6 \mathrm{MM}$, MECHANICAL SCANNING LENGTH OF $0.3 \mathrm{M}$ WITH INTERVAL $d v=4 \mathrm{MM})$.

\begin{tabular}{ccc}
\hline Parameters & Config. 1 & Config.2 \\
\hline TR Separation $(\mathrm{mm})$ & $\mathrm{S}_{\mathrm{TR}}=181$ & $\mathrm{~S}_{\mathrm{TR}}=181$ \\
Element Pattern & Wide & Narrow \\
Target Distance & $1.1 \mathrm{~m}$ & $1.4 \mathrm{~m}$ \\
Resolution by $(3)(\mathrm{mm} \times \mathrm{mm})$ & $3.91 \times 2.50$ & $4.97 \times 3.18$ \\
Subtended angle $\langle x, y\rangle$ & $\left\langle 9.98^{\circ}, 15.53^{\circ}\right\rangle$ & $\left\langle 7.85^{\circ}, 12.23^{\circ}\right\rangle$ \\
Resolution by $(2)(\mathrm{mm} \times \mathrm{mm})$ & $3.92 \times 2.52$ & $4.98 \times 3.26$ \\
Image SNR $(\mathrm{ISNR})$ & $15.85 \mathrm{~dB}$ & $23.11 \mathrm{~dB}$ \\
\hline
\end{tabular}

Wide: HPBW of about $42^{\circ}$ with a peak gain of $12.95 \mathrm{~dB}$ at $220 \mathrm{GHz}$; Narrow: HPBW of about $12^{\circ}$ with a peak gain of $23.68 \mathrm{~dB}$ at $220 \mathrm{GHz}$.

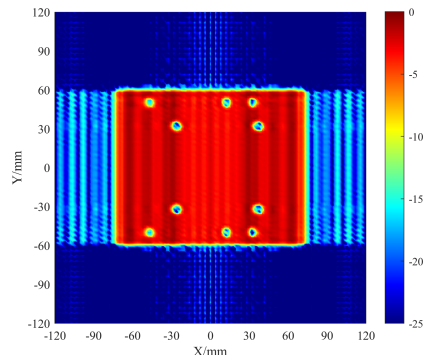

(a)

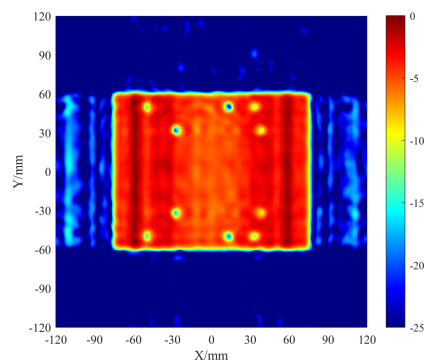

(c)

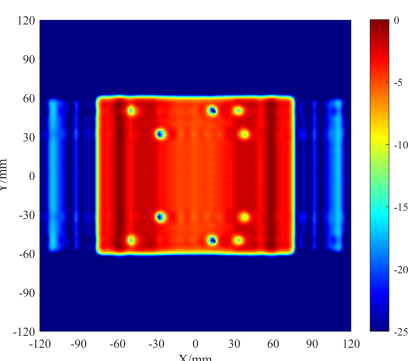

(b)

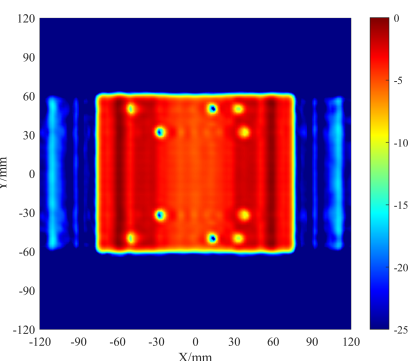

(d)
Fig. 6. Reconstructed single-pass synthetic aperture focusing images based on full simulated data (a) configuration 1 (ISNR $=15.85 \mathrm{~dB}$ ) (b) configuration 2 (ISNR=23.11 dB) and LRMC data of configuration 2 with (c) $40 \%$ (ISNR=17.38 dB) (d) 45\% (ISNR=21.44 dB) sampled simulated data.

SNRs are calculated in Table III. The MIMO array in simulation consists of $8 \mathrm{Tx}$ elements and $8 \mathrm{Rx}$ elements in which the Tx element spacing $d t$ is $6 \mathrm{~mm}$. The mechanical scanning length is $0.3 \mathrm{~m}$ and the interval $d v$ between sampling points is $4 \mathrm{~mm}$. This sampling setting provides a FOV (field of view) of $0.192 \mathrm{~m} \times 0.3 \mathrm{~m}$. Two kinds of array elements with a wide beam $\left(42^{\circ} \mathrm{HPWB}\right.$ and peak gain of $\left.12.95 \mathrm{~dB}\right)$ or narrow beam $\left(12^{\circ} \mathrm{HPBW}\right.$ and peak gain of $23.68 \mathrm{~dB}$ modelling the standard WR3 horn used in experiments) are compared in simulation. The theoretical resolutions along two directions can be roughly estimated by (3) or by equation (2) for a non-aperture limited imaging system. Tx array and Rx array are separated by $S_{\mathrm{TR}}$ $=181 \mathrm{~mm}$ to imitate the experimental set-up, which causes more artifacts in background and deterioration on image SNR [25]. When the array element with narrow beam width of high gain is used, the image SNR is improved. More analyses and simulation of various scenarios can be referred to our previous 
This article has been accepted for publication in a future issue of this journal, but has not been fully edited. Content may change prior to final publication. Citation information: DOI: 10.1109/TMTT.2021...

IEEE TRANSACTIONS ON MICROWAVE THEORY AND TECHNIQUES, VOL. XX, NO. XX, XXXX 2021

studies [25], [46]. It is demonstrated that the computational analysis in MATLAB provides an effective approach to design the imaging scheme including array configuration, scanning aperture and mechanical sampling interval according to the desired practical imaging performance.

Furthermore, we tested the proposed LRMC sparse imaging algorithm only based on the simulated data of configuration 2 , which is applied in proof-of-concept experiments. $40 \%$ and $45 \%$ sparse sampled data are used to retrieve the approximation of full sampling matrix and then reconstruct the target images, as shown in Fig.6(c) and Fig.6(d), respectively. It is worth mentioning that all these percentages ( without referring to Nyquist $\lambda / 2$ sampling ) in this paper are calculated based on their corresponding reference data that is already a significant data reduction compared to Nyquist sampling data with halfwavelength $-\lambda / 2$ interval. For example, the intervals along two directions in simulation are $3 \mathrm{~mm}$ and $4 \mathrm{~mm}$. The generated full data as reference is only $3.87 \%$ of Nyquist sampling scheme with $\lambda / 2$ sample interval. The results show that $40 \%$ data almost reconstruct the target image perfect except for more artifacts in background and little deformation on edges. When $45 \%$ data is used, the target image shows no visible difference from the reference and the image SNR achieves $21.44 \mathrm{~dB}$, which is higher than the $17.38 \mathrm{~dB}$ of $40 \%$ data used and comparable to the $23.11 \mathrm{~dB}$ in reference. Thus, the proposed SVT LRMC sparse imaging algorithm has been preliminary verified feasible in simulation.

\section{Proof-OF-ConcePt ExPERIMENTS ANd Results}

The proof-of-concept experimental set-up is shown in Fig.7. Two THz mixer heads highlighted in blue are mounted on two linear horizontal scanning stages moving to each $\mathrm{Tx}$ and $\mathrm{Rx}$ element's positions, imitating the $\mathrm{Tx}$ and $\mathrm{Rx}$ array of SPA respectively. Since the scanning stages are fixed, the target mounted on the head of PNF-XYV- $0.9 \mathrm{~m} \times 0.9 \mathrm{~m}$ scanning system moves vertically to inversely imitate a relatively mechanical scanning of the SPA to a fixed target. Besides, it changes $\mathrm{N}$ positions along horizontal (electronic scanning) direction to realize the N-pass movements of SPA inversely. Fig. 8 shows the set-up of the whole system and the target under test mounted on the scanning system. The system under test uses a SPA consisting of $\mathrm{Nt}=8 \mathrm{Tx}$ elements and $\mathrm{Nr}=$ $8 \mathrm{Rx}$ elements, the Tx element spacing $d t$ is $6 \mathrm{~mm}$ so the electronic sampling interval is $3 \mathrm{~mm}$. The horns utilized in the experiment are WR3 $(220 \sim 325 \mathrm{GHz})$ standard pyramidal horns with a peak gain of about $23.81 \mathrm{~dB}$ at $220 \mathrm{GHz}$ and the typical output power from the $\mathrm{THz}$ mixer at $220 \mathrm{GHz}$ is -13 $\mathrm{dBm}(0.05 \mathrm{~mW})$ [25].

\section{A. Pure Metallic Target with Smooth Surface}

The target is a rectangular metallic plate $(140 \mathrm{~mm} \times 120$ $\mathrm{mm} \times 5 \mathrm{~mm}$ ) having 10 holes with diameters ranging from $5 \mathrm{~mm}$ to $8 \mathrm{~mm}$ and the scanning length (width of field of view) is $192 \mathrm{~mm}$. The mechanical scanning length is $0.3 \mathrm{~m}$ with an interval $d v$ of $4 \mathrm{~mm}$, and two experiments of target at $1.10 \mathrm{~m}$ and $1.40 \mathrm{~m}$ from the scanning plane are carried out.

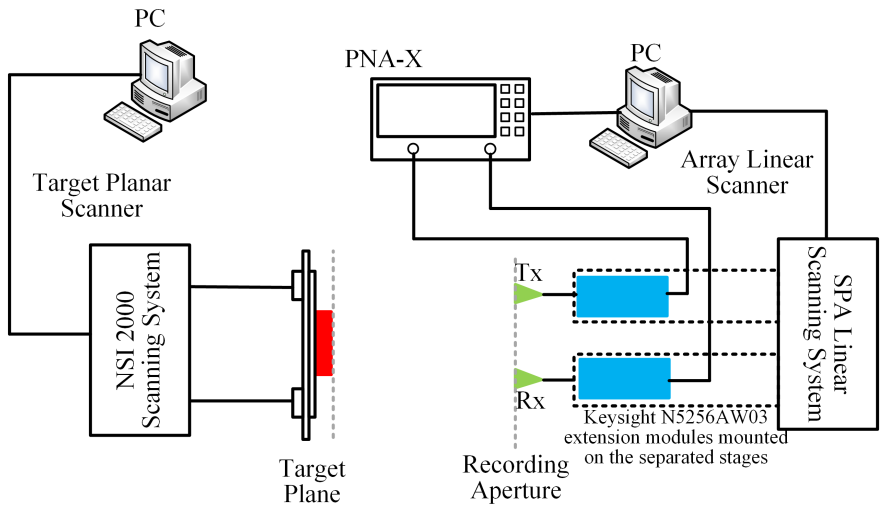

Fig. 7. Schematic of the proposed experimental imaging system in the lab.

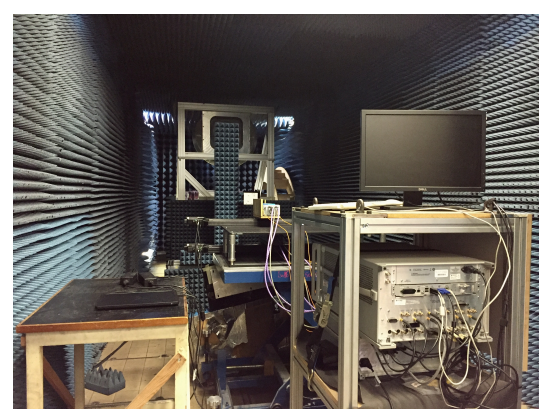

(a)

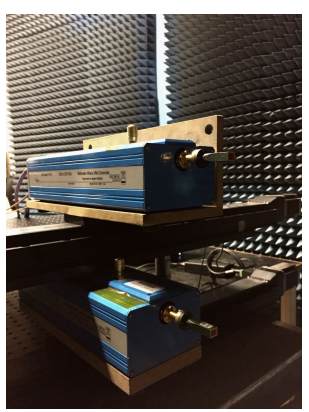

(b)

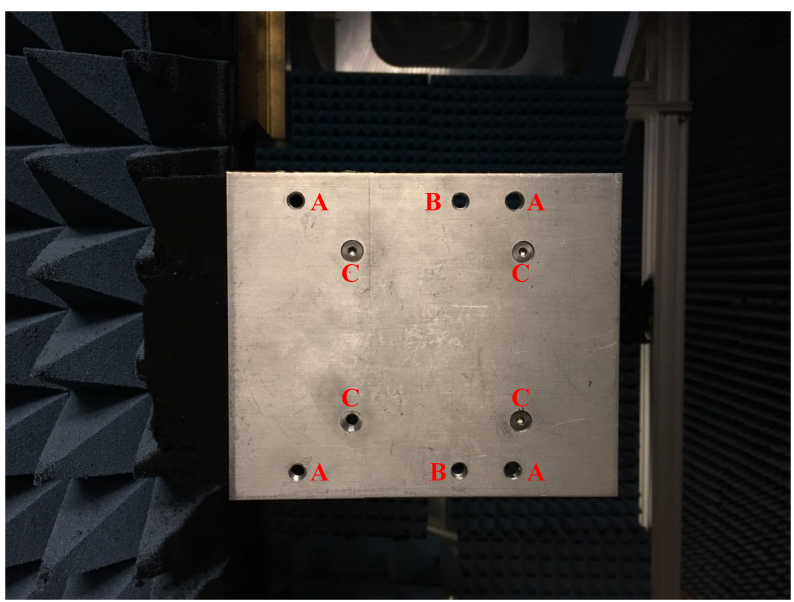

(c)

Fig. 8. The photographs of the proof-of-concept experimental set-up.(a) whole view of the system (b) scanning stages (c) close view of the target mounted on the PNF-XYV-0.9m $\times 0.9 \mathrm{~m}$ scanning system.

The number of different passes $\mathrm{N}$ is 4 and the step along $\mathrm{x}$ axis is $20 \mathrm{~mm}$. The current sampling configuration makes even total 4 passes data only occupies $15.50 \%$ of 1-pass Nyquist $\lambda / 2$ sampling data. Fig.9 and Fig.10 show the reconstructed single-pass synthetic focusing images of two experiments. The corresponding multi-pass interferometric synthetic aperture images have been shown in Fig.11. There are 10 holes that can be identified in Fig.11 that is more than any image in Fig.9 and Fig.10. In addition, the artifacts in background (blue area) are less while the target image (red area) is more uniform, so the 
image SNR is obviously improved, as indicated in Fig.12. It is worth noting that the image SNRs in the second experiment of target placed at $1.4 \mathrm{~m}$ are higher due to targets within FOV defined as $0.5 \cdot N t \cdot N r \cdot d t$ and widened beam-width with propagation [25].

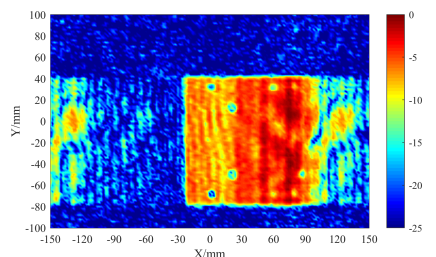

(a)

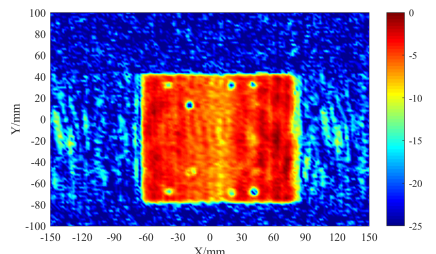

(c)

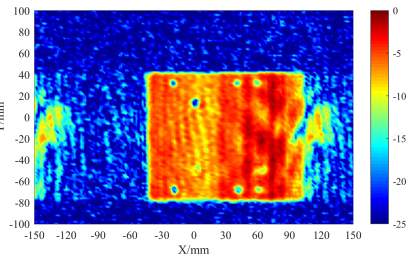

(b)

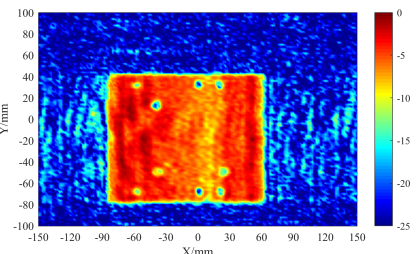

(d)
Fig. 9. Reconstructed single-pass synthetic aperture focusing images of target at (a) position $\times 1$ (b) position $\times 2(x 1-20 \mathrm{~mm})(\mathrm{c})$ position $\times 3(\mathrm{x} 1-40 \mathrm{~mm})(\mathrm{d})$ position $\mathrm{x} 4$ (x1-60 mm).

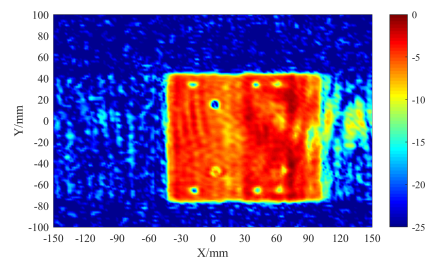

(a)

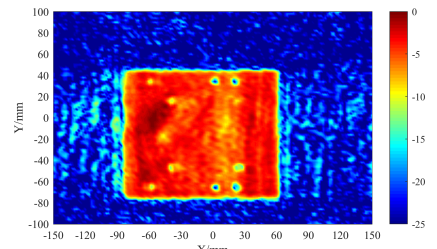

(c)

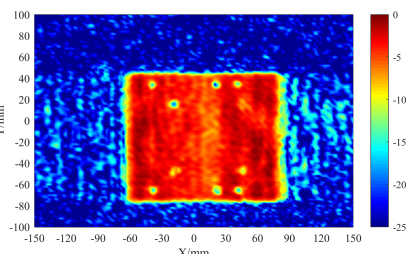

(b)

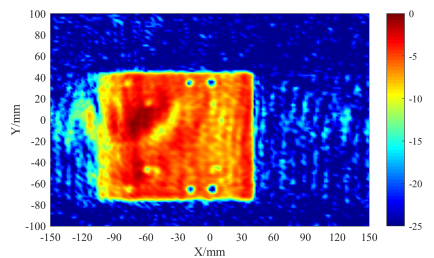

(d)
Fig. 10. Reconstructed single-pass synthetic aperture focusing images of target at (a) position $\mathrm{x} 1$ (b) position $\mathrm{x} 2(\mathrm{x} 1-20 \mathrm{~mm})$ (c) position $\mathrm{x} 3$ (x1-40 $\mathrm{mm})(\mathrm{d})$ position $\mathrm{x} 4(\mathrm{x} 1-60 \mathrm{~mm})$.

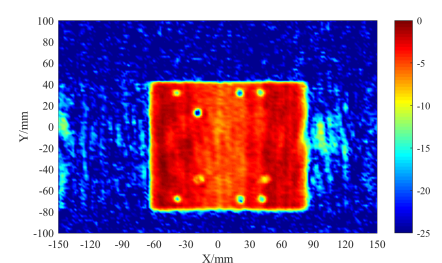

(a)

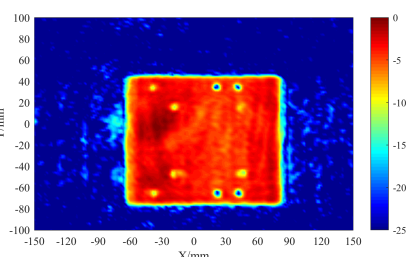

(b)
Fig. 11. Reconstructed 4-pass interferometric synthetic aperture focusing images of pure metallic target at (a) $1.1 \mathrm{~m}$ (b) $1.4 \mathrm{~m}$.

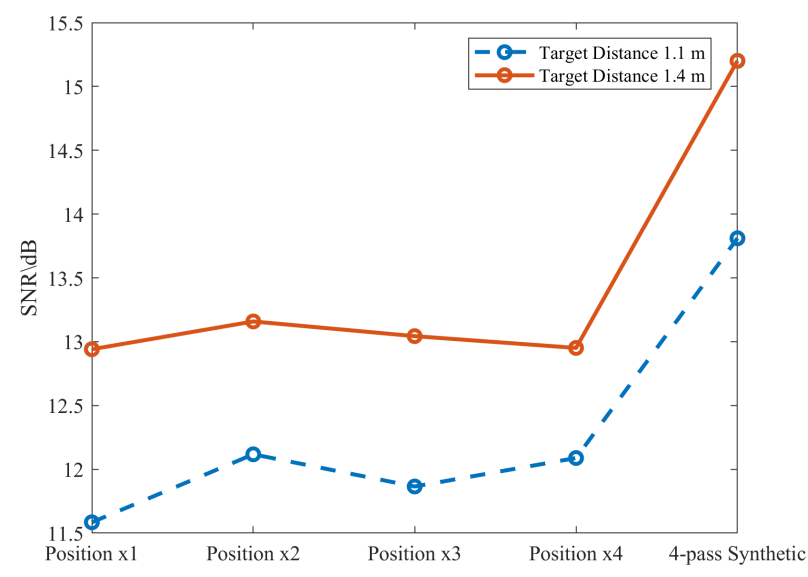

Fig. 12. Image SNRs of pure metallic target in two experiments.

\section{B. Complex Target including Metallic Pattern and Dielectric Substrate}

In addition to the pure metallic target, the more challenging target of metallic patches etched on the dielectric substrate $(260 \mathrm{~mm} \times 180 \mathrm{~mm} \times 1.6 \mathrm{~mm})$ imitating the mannequin as shown Fig. 13 have been tested. The target distance is $1.40 \mathrm{~m}$ and mechanical scanning length is $0.4 \mathrm{~m}$ with an interval of 2.5 $\mathrm{mm}$, so the theoretical resolutions are $4.98 \mathrm{~mm}$ and $3.26 \mathrm{~mm}$ along horizontal and vertical directions according to (2). There are $N=5$ different passes with a step of $10 \mathrm{~mm}$ along x-axis. The current sampling configuration makes even total 5 passes data only occupies $31.00 \%$ of 1-pass Nyquist $\lambda / 2$ sampling data. The reconstructed single-pass images of the target have been shown in Fig.14(a) to Fig.14(e). The corresponding image SNRs as shown in Fig.15 range from $10.69 \mathrm{~dB}$ to $11.01 \mathrm{~dB}$. The artifacts in the background are high while high power (red area) spreads on the non-metallic surface, which degrades image contrast indicated by low SNRs. It results from two factors. One is lower reflectively difference between metal and dielectric substrate. The other one is deteriorating focusing caused by much more scattering from the uneven surface of dielectric substrate. Thus, the proposed multi-pass interferometric synthetic aperture focusing technique is more desired to significantly improve the image quality and target identification. It has been verified in Fig.14(f) and the image SNR improves by about $4.06 \mathrm{~dB}$.

\section{Image Reconstruction of Random Under-sampled Sparse Data with LRMC}

Although the proposed multi-pass interferometric synthetic aperture focusing imaging helps to improve the image quality, it increases the sampling data/time. In addition, if we implement full planar electronic scanning system, the number of transceivers and hardware associated to samples are increased too. This will increase the system cost. Thus, we proposed to use random sparse under-sampling to reduce the number and LRMC technique to complete the missing samples, reconstructing the image on the basis of the simplified SVT algorithm in this paper. We only use the experimental data of more challenging target as a study case for brevity. 50\% and 


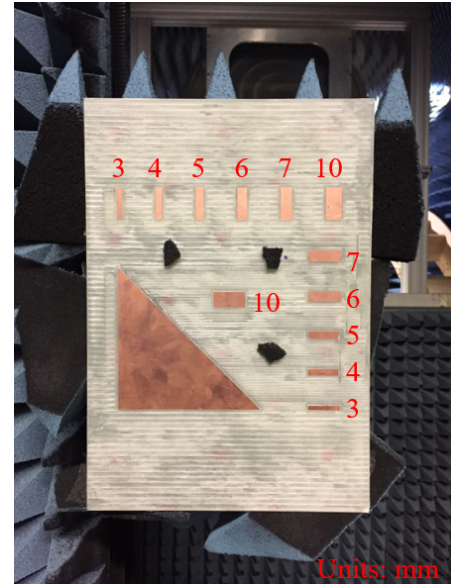

Fig. 13. The photographs of the experimental complex target including metallic pattern and dielectric substrate.

$66.6 \%$ data of each single-pass scanning are investigated, this leads to $84.5 \%$ and $79.3 \%$ reduction compared to Nyquist $\lambda / 2$ sampling data.

The reconstructed images are shown in Fig.16(a) 16(b), respectively. And the image SNRs are listed in Table IV. As implied from the results, it is reasonable that more data will improve the image SNR, that is why the references have the highest image SNR. However, reconstructed images based on $66.6 \%$ data already show no visible difference from the references.

In order to demonstrate reducing the number of transceivers and related system cost, some of Tx/Rx elements in the SPA in each mechanical scanning position are randomly removed thus random sparse sampling is achieved. There must be more approaches to designing optimal "sparse" sampling patterns or aperiodic sparse arrays, which is not the focus of this paper so it will not be discussed due to limited pages. According to Table I, the number of $M t$ Tx elements removed will cause samples missed one following one with an interval of $N t / 2$ samples while the number of $M r \mathrm{Rx}$ elements removed will cause samples missed one block,each block consist of $N t / 2$ samples, following one block with an interval of $N r$ blocks. If both Tx and Rx elements are removed, there will be $N t \cdot N r$ Tx-Rx pairs overlapped so total of $M t \cdot N r+M r \cdot N t-N t$. $N r$ samples are missed. For example, 17(a) shows a sparse sampling pattern with $1 \mathrm{Tx}$ and $1 \mathrm{Rx}$ elements removed in each row. Fig.17(b) 17(d) show the reconstructed multi-pass images with 3 Tx \& $3 \mathrm{Rx}, 2 \mathrm{Tx} \& 2 \mathrm{Rx}$ and $1 \mathrm{Tx} \& 1 \mathrm{Rx}$ removed. The images SNRs are compared in Table IV. The results have shown that the scenario of $5 \mathrm{Tx} \& 5 \mathrm{Rx}$ left $(3$ Tx \& $3 \mathrm{Rx}$ removed) is capable of providing an improvement than single-pass image. This improvement increases with more transceivers/data used so the scenario of $2 \mathrm{Tx} \& 2 \mathrm{Rx}$ removed (56.25\% and above data) is promising to achieve equivalent image quality to reference, this corresponds to $82.51 \%$ more data reduction compared to $\lambda / 2$ sampling. The scenario of 1 Tx \& $1 \mathrm{Rx}$ removed provides $0.125 \mathrm{~dB}$ higher image SNR that, in addition to deviation, possibly benefits from random sampling patter that is helpful to suppress the artifacts.

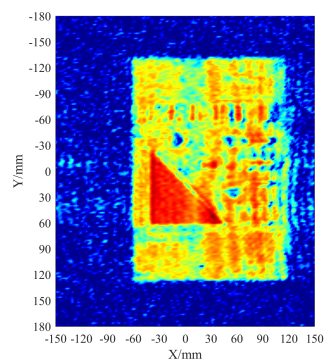

(a)

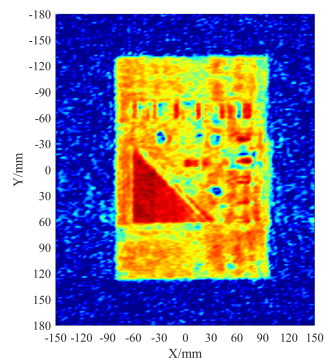

(c)

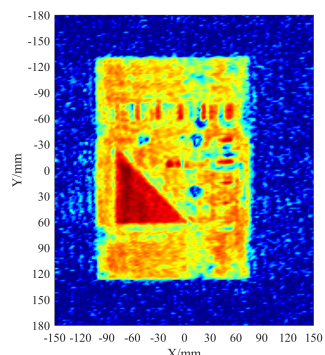

(e)
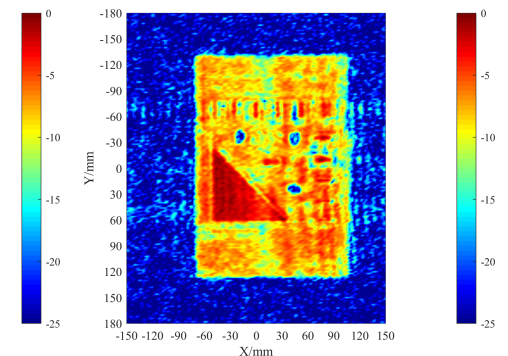

(b)
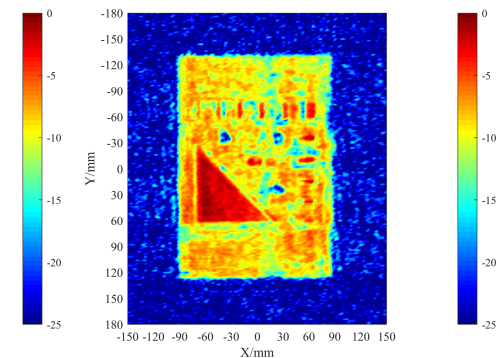

(d)
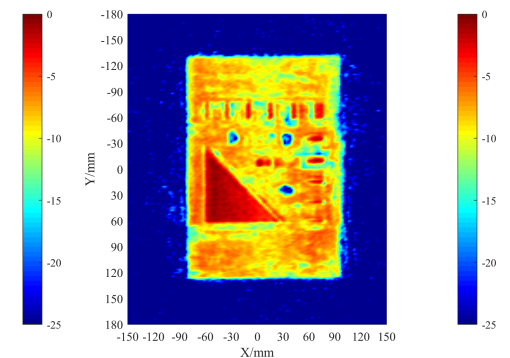

(f)
Fig. 14. Reconstructed single-pass synthetic aperture focusing images in experiment D2 of target at (a) position x1 (b) position x2(x1-10 mm) (c) position $\times 3(\times 1-20 \mathrm{~mm})(\mathrm{d})$ position $\times 4(\mathrm{x} 1-30 \mathrm{~mm})$ (e) position $\times 5(\mathrm{x} 1-40$ $\mathrm{mm}$ ) and (f) corresponding 5-pass interferometric synthetic aperture focusing image.

TABLE IV

COMPARISON OF IMAGE SNR WITH SPARSELY APPROXIMATED $S_{f u l l}$ AND EXACT $S_{\text {full }}$.

\begin{tabular}{ccc}
\hline Parameters & Sampling Scheme & Image SNR / dB \\
\hline Samples & $50 \%$ sampled data & 12.538 (Fig.16(a)) \\
removed & $66.6 \%$ sampled data & 13.689 (Fig.16(b)) \\
& 3 Tx \& 3 Rx / 39.06\% data & 11.849 (Fig.17(b)) \\
Tx \& Rx & 2 Tx \& 2 Rx / 56.25\% data & 13.315 (Fig.17(c)) \\
removed & 1 Tx \& 1 Rx / 76.56\% data & 14.195 (Fig.17(d)) \\
& 5-pass 100\% data w/o LRMC & 14.07 (Fig.14(f)) \\
\hline
\end{tabular}

The percentages are calculated on the basis of Reference- $S_{f u l l}$ data that is only $31.10 \%$ of 1 -pass Nyquist $\lambda / 2$ sampling data.

\section{DISCUSSION}

In proof-of-concept experiments, it is demonstrated that the single-pass synthetic aperture imaging with large element spacing (such as $4.40 \lambda$ in this paper that leads to significant data reduction of $\lambda / 2$ sampling ) is capable of identifying pure metallic targets, which saves the data acquisition time and system cost. The proposed multi-pass interferometric synthetic aperture sparse imaging technique improves the image quality 


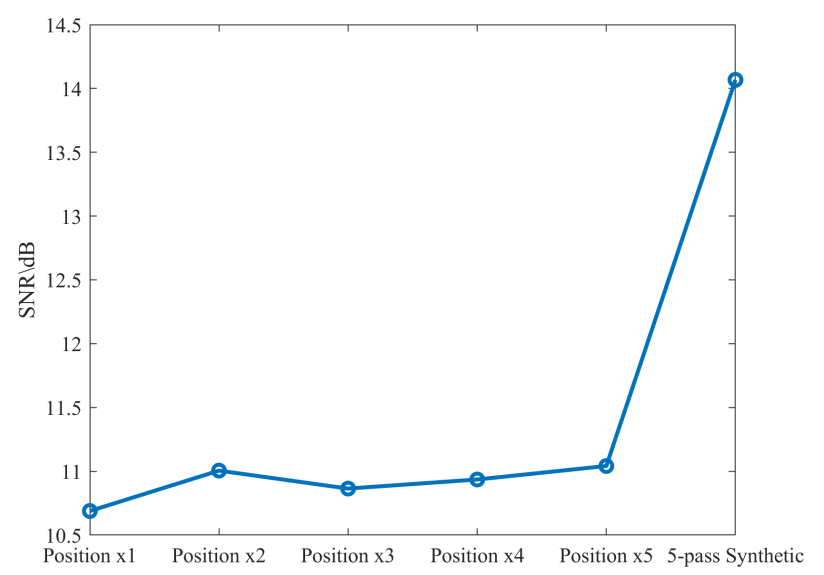

Fig. 15. Image SNRs of complex targets consisting of metallic pattern and dielectric substrate.

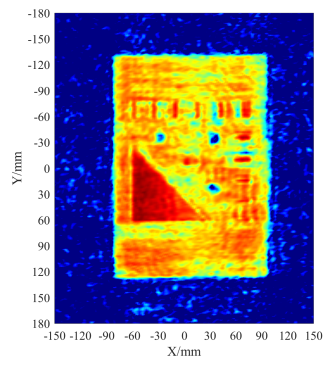

(a)
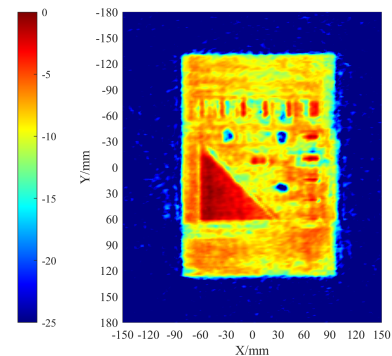

(b)
Fig. 16. Reconstructed multi-pass synthetic aperture focusing images with LRMC-SVT retrieved data based on (a) $50 \%$ (b) $66.6 \%$ sampled data.

but maintain the low sampling data ratio, which works as a complementary mode for more challenging targets. The reconstruction based on LRMC technique has demonstrated acceptable image quality in both single-pass synthetic aperture focusing image and multi-pass interferometric synthetic aperture focusing image. Therefore, the simplified operating procedures with a constant $d s$ using such an advance imaging system for target detection can be summarized as follows:

1) Start, initialise $n=1$, step value $d s$ and pass number $N$ generated accordingly;

2) Scan and record $n$ th-pass under sampled dataset;

3) Reconstruct $n$th single-pass synthetic aperture focusing image and if $n>1$, reconstruct $n$-pass interferometric synthetic aperture focusing image simultaneously;

4) If target is identified, go to step 5) so cease the detection earlier, else if $n<=N, n=n+1$, go to step 2);

5) End (successful target detection).

We admit that more effort might be demanded to make full use of the proposed imaging system, such as designing optimal pass step $d s$, other potential 1D/2D sparse MIMO arrays or sampling patterns. Therefore, we hope more research and improvements will be followed up by either the authors or readers in the future.

\section{CONCLUSION}

A systematic method of computational imaging simulation for target detection has been proposed. It not only leads to

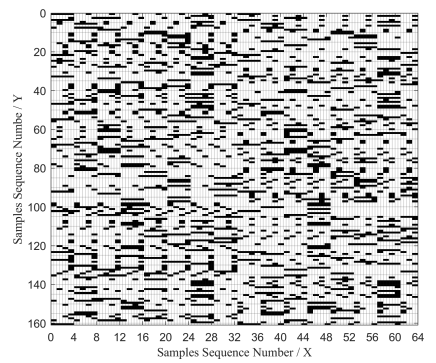

(a)

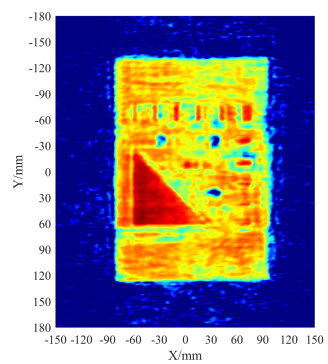

(c)

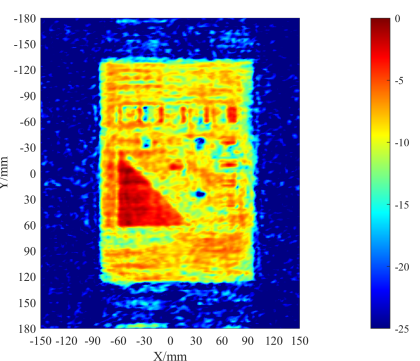

(b)

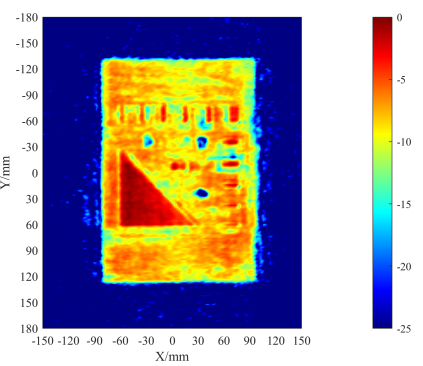

(d)
Fig. 17. (a) Example of sparse sampling pattern with $1 \mathrm{Tx} \& 1 \mathrm{Rx}$ randomly removed in each row(white:samples) and reconstructed multi-pass synthetic aperture focusing images with LRMC-SVT method when (b) 3 Tx \& 3 Rx removed (c) 2 Tx \& 2 Rx removed (d) 1 Tx \& 1 Rx removed.

designing the sampling scheme for an imaging system but also provides an effective evaluation on the practical imaging performance.

The proposed $\mathrm{THz}$ MIMO sparse imaging scheme using mutli-pass interferometric synthetic aperture focusing technique and LRMC technique has been verified in both simulation and proof-of-concept experiments. The multi-pass interferometric synthetic aperture focusing technique allows to improve the imaging performance without adding additional transceivers/channels. The LRMC technique enables to reduce the sampling time and system cost associated to transceivers but without compromising on the high image quality.

An novel dual-mode operation has also been proposed so they can operate simultaneously with help of real-time imaging algorithm. The image quality is increasing as new pass interferometric dataset is added. Also, the scanning and image reconstruction will cease earlier once the target is identified in either of two kinds of results, which is effective than directly using traditional $\lambda / 2$ sampling scheme.

\section{ACKNOWLEDGMENT}

Authors would like to thank Dr Debbie Fellows and Torben Voigt from Altair Engineering for helpful technical discussions on imaging simulation with $\mathrm{FEKO}^{\mathrm{TM}}$.

\section{REFERENCES}

[1] S. S. Ahmed, "Microwave imaging in security-two decades of innovation," IEEE J. Microwaves, vol. 1, no. 1, pp. 191-201, Jan. 2021.

[2] Y. Zhang, X. R. Jiang, Z. Y. Ma, Q. Yang, Y. Zeng, B. Deng, and H. Q. Wang, "An efficient interpolation-free algorithm for cylindrical millimeter-wave holographic three-dimensional reconstruction," Microw. Opt. Technol. Lett., vol. 63, no. 4, pp. 1018-1023, Sep. 2020. 
[3] O. Yurduseven, D. L. Marks, J. N. Gollub, and D. R. Smith, "A reconfigurable millimeter-wave spotlight metasurface aperture integrated with a frequency-diverse microwave imager for security screening," in Proc. SPIE 10634, Passive and Active Millim. Wave Imag. XXI, vol. 10634. International Society for Optics and Photonics, May 2018, p. 1063406.

[4] N. A. Salmon, "Indoor full-body security screening: Radiometric microwave imaging phenomenology and polarimetric scene simulation," IEEE Access, vol. 8, pp. 144621-144637, Aug. 2020.

[5] F. Gumbmann and A. Schiessl, "Short-range imaging system with a nonuniform sfcw approach," IEEE Transactions on Microwave Theory and Techniques, vol. 65, no. 4, pp. 1345-1354, Apr. 2017.

[6] B. Liang, X. Shang, X. Zhuge, and J. Miao, "Accurate near-field millimeter-wave imaging of concave objects-a case study of dihedral structures under monostatic array configurations," IEEE Trans. Geosci. Remote Sens., vol. 58, no. 5, pp. 3469-3483, May 2020.

[7] J. F. Federici, B. Schulkin, F. Huang, D. Gary, R. Barat, F. Oliveira, and D. Zimdars, "Thz imaging and sensing for security applications-explosives, weapons and drugs," Semicond. Sci. Technol., vol. 20, no. 7, pp. S266-S280, Jun. 2005.

[8] D. A. Robertson et al., "High resolution, wide field of view, real time 340ghz 3d imaging radar for security screening," in Proc. SPIE 10189, Passive and Active Millim. Wave Imag. XX, vol. 10189. International Society for Optics and Photonics, May 2017, p. 101890C.

[9] D. A. Robertson et al., "A high frame rate, $340 \mathrm{ghz} 3 \mathrm{~d}$ imaging radar for security," in 2018 IEEE Radar Conf. (RadarConf18), Jun. 2018, pp. 0055-0060.

[10] R. Li, C. Li, H. Li, S. Wu, and G. Fang, "Study of automatic detection of concealed targets in passive terahertz images for intelligent security screening," IEEE Trans. Terahertz Sci. Technol., vol. 9, no. 2, pp. 165176, Dec. 2019

[11] S. Rowe et al., "A passive terahertz video camera based on lumped element kinetic inductance detectors," Rev. Sci. Instrum., vol. 87, no. 3, p. 033105, Mar. 2016

[12] H. Feng et al., "A passive video-rate terahertz human body imager with real-time calibration for security applications," Appl. Phys. B, vol. 126, no. 8, pp. 1-9, Aug. 2020.

[13] E. Heinz, T. May, D. Born, G. Zieger, S. Anders, V. Zakosarenko, H.G. Meyer, and C. Schäffel, "Passive 350 ghz video imaging systems for security applications," J. Infrared Millim. Terahertz Waves, vol. 36, no. 10, pp. 879-895, Jun. 2015.

[14] Y. Meng, A. Qing, C. Lin, J. Zang, Y. Zhao, and C. Zhang, "Passive millimeter wave imaging system based on helical scanning," Sci. Rep, vol. 8, no. 1, pp. 1-12, May 2018.

[15] Y. Cheng, L. Qiao, D. Zhu, Y. Wang, and Z. Zhao, "Passive polarimetric imaging of millimeter and terahertz waves for personnel security screening," Opt. Lett., vol. 46, no. 6, pp. 1233-1236, Feb. 2021.

[16] Y. Zhou, J. Yu, L. Pan, Y. Wang, and Q. Cao, "3d mimo imaging architecture for walk through personnel security screening," IET Microw. Antennas Propag., vol. 12, no. 14, pp. 2206-2212, Sep. 2018.

[17] S. Li, S. Wang, Q. An, G. Zhao, and H. Sun, "Cylindrical mimo array based near-field microwave imaging," IEEE Trans. Antennas Propag., vol. 69, no. 1, pp. 612-617, Jan. 2021.

[18] D. M. Sheen and T. E. Hall, "Reconstruction techniques for sparse multistatic linear array microwave imaging," in Proc. SPIE9078, Passive and Active Millim. Wave Imag. XVII, vol. 9078, Jun. 2014, p. 90780I.

[19] S. S. Ahmed, A. Schiessl, and L.-P. Schmidt, "A novel fully electronic active real-time imager based on a planar multistatic sparse array," IEEE Trans. Microw. Theory Techn., vol. 59, no. 12, pp. 3567-3576, Dec. 2011.

[20] L. Ding, S. Wu, X. Ding, P. Li, and Y. Zhu, "Off-the-grid sparse imaging by one-dimensional sparse mimo array," IEEE Sensors J., vol. 18, no. 24 pp. 9993-10001, Oct. 2018.

[21] F. Gumbmann and L.-P. Schmidt, "Millimeter-wave imaging with optimized sparse periodic array for short-range applications," IEEE Trans. Geosci. Remote Sens., vol. 49, no. 10, pp. 3629-3638, Oct. 2011.

[22] B. Cheng et al., "340-ghz 3-d imaging radar with 4tx-16rx mimo array," IEEE Trans. Terahertz Sci. Technol., vol. 8, no. 5, pp. 509-519, Sep. 2018.

[23] R. Zhu, J. Zhou, G. Jiang, B. Cheng, and Q. Fu, "Grating lobe suppression in near range mimo array imaging using zero migration," IEEE Trans. Microw. Theory Techn., vol. 68, no. 1, pp. 387-397, Jan. 2020.

[24] H. Gao, C. Li, S. Wu, H. Geng, S. Zheng, X. Qu, and G. Fang, "Study of the extended phase shift migration for three-dimensional mimo-sar imaging in terahertz band," IEEE Access, vol. 8, pp. 24 773-24 783, Jan. 2020 .
[25] S. Hu, C. Shu, Y. Alfadhl, and X. Chen, "A thz imaging system using linear sparse periodic array," IEEE Sensors J., vol. 20, no. 6, pp. 32853292, Mar. 2019

[26] S. Hu, C. Shu, Y. Alfadhl, X. Chen, and K. Wang, "Thz personnel screening system with multi-pass interferometric synthetic aperture technique," in Proc. IEEE UK-Europe-China Workshop Millim. Waves and Terahertz Technol.(UCMMT), Dec. 2020, pp. 1-3.

[27] G. Fornaro, F. Lombardini, and F. Serafino, "Three-dimensional multipass sar focusing: Experiments with long-term spaceborne data," IEEE Trans. Geosci. Remote Sens., vol. 43, no. 4, pp. 702-714, Apr. 2005.

[28] J. Homer, I. Longstaff, Z. She, and D. Gray, "High resolution 3-d imaging via multi-pass sar," IEE Proc. Radar, Sonar and Navigation, vol. 149 , no. 1, pp. 45-50, Feb. 2002.

[29] M. Schmitt and U. Stilla, "Adaptive multilooking of airborne single-pass multi-baseline insar stacks," IEEE Trans. Geosci. Remote Sens., vol. 52, no. 1, pp. 305-312, Feb. 2013.

[30] M.-H. Ka, P. E. Shimkin, A. I. Baskakov, and M. I. Babokin, "A new single-pass sar interferometry technique with a single-antenna for terrain height measurements," Remote Sens., vol. 11, no. 9, p. 1070, Mar. 2019.

[31] F. Salvetti, M. Martorella, E. Giusti, and D. Staglianò, "Multiview three-dimensional interferometric inverse synthetic aperture radar," IEEE Trans. Aerosp. Electron. Syst., vol. 55, no. 2, pp. 718-733, Apr. 2019.

[32] B. Tian, Z. Lu, Y. Liu, and X. Li, "Review on interferometric isar 3d imaging: Concept, technology and experiment," Signal Process., vol. 153, pp. 164-187, Dec. 2018.

[33] S. Hu, C. Shu, Y. Alfadhl, and X. Chen, "Thz sparse periodic array imaging system using compressed sensing," IET Microw. Antennas Propag., vol. 14, no. 11, pp. 1157-1161, Jul. 2020.

[34] S. Wu, L. Ding, P. Li, Y. Li, L. Chen, and Y. Zhu, "Millimeter-wave sar sparse imaging with 2-d spatially pseudorandom spiral-sampling pattern," IEEE Trans. Microw. Theory Techn., vol. 68, no. 11, pp. 46724683, Nov. 2020

[35] S. Bernhardt, R. Boyer, S. Marcos, and P. Larzabal, "Compressed sensing with basis mismatch: Performance bounds and sparse-based estimator," IEEE Trans. Signal Process., vol. 64, no. 13, pp. 3483-3494, Jul. 2016.

[36] H. Bi, C. Jiang, B. Zhang, Z. Wang, and W. Hong, "Radar change imaging with undersampled data based on matrix completion and bayesian compressive sensing," IEEE Geosci. Remote Sens. Lett., vol. 12, no. 7, pp. 1546-1550, Jul. 2015.

[37] J.-F. Cai, E. J. Candès, and Z. Shen, "A singular value thresholding algorithm for matrix completion," SIAM J. optim., vol. 20, no. 4, pp. 1956-1982, Mar. 2010.

[38] L. T. Nguyen, J. Kim, and B. Shim, "Low-rank matrix completion: A contemporary survey," IEEE Access, vol. 7, pp. $94215-94237$, Jul. 2019.

[39] D. Yang, G. Liao, S. Zhu, X. Yang, and X. Zhang, "Sar imaging with undersampled data via matrix completion," IEEE Trans. Geosci. Remote Sens., vol. 11, no. 9, pp. 1539-1543, Sep. 2014.

[40] S. Zhang, G. Dong, and G. Kuang, "Matrix completion for downwardlooking 3-d sar imaging with a random sparse linear array," IEEE Trans. Geosci. Remote Sens., vol. 56, no. 4, pp. 1994-2006, Apr. 2018.

[41] W. Si, X. Zhuge, and J. Miao, "Sparse nonuniform frequency sampling for fast sfcw radar imaging," IEEE Access, vol. 8, pp. 126573-126581, Jul. 2020.

[42] R. Zhu, J. Zhou, B. Cheng, Q. Fu, and G. Jiang, "Sequential frequencydomain imaging algorithm for near-field mimo-sar with arbitrary scanning paths," IEEE J. Sel. Topics Appl. Earth Observ. Remote Sens., vol. 12 , no. 8, pp. 2967-2975, Aug. 2019.

[43] A. Tamminen, J. Ala-Laurinaho, and A. Räisänen, "Indirect holographic imaging: evaluation of image quality at $310 \mathrm{ghz}$," in Proc. SPIE 7670, Passive Millim. Wave Imag. Technol. XIII, vol. 7670. International Society for Optics and Photonics, Apr. 2010, p. 76700A.

[44] U. Jakobus, "Overview of hybrid methods in feko: Theory and applications," in Proc. 2010 Int. Conf. Electromagn. Adv. Appl., Dec. 2010, pp. 434-437.

[45] A. G. Aguilar, J. van Tonder, U. Jakobus, and F. Illenseer, "Overview of recent advances in the electromagnetic field solver feko," in Eur. Conf. Antennas Propag. (EuCAP), Aug. 2015, pp. 1-5.

[46] S. Hu, "Study on thz imaging system for concealed threats detection," $\mathrm{Ph} . \mathrm{D}$. dissertation, Queen Mary University of London, London, Dec. 2020.

[47] E. J. Candès and T. Tao, "The power of convex relaxation: Near-optimal matrix completion," IEEE Trans. Inf. Theory, vol. 56, no. 5, pp. 20532080, May 2010.

[48] E. J. Candès, X. Li, Y. Ma, and J. Wright, "Robust principal component analysis?" J. ACM, vol. 58, no. 3, pp. 1-37, May 2011. 


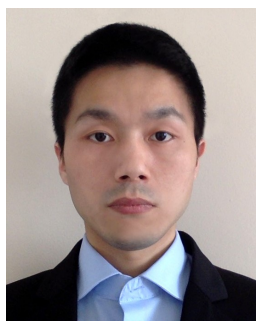

Shaoqing Hu (M’20) was born in Guzhen, Anhui Province, China in 1992. He received the BEng and MEng degrees from the University of Electronic Science and Technology of China, Chengdu, Sichuan, China, in 2013 and 2016, and the Ph.D. degree from Queen Mary University of London, London, UK, in 2020.

He is currently a lecturer with the Department of Electronic and Electrical Engineering, College of Engineering, Design \& Physical Sciences, Brunel University London. He is the author of more than 10 articles. His research interests include antennas, arrays, compressed sensing, millimeter wave and $\mathrm{THz}$ sparse-aperture imaging.

Dr Hu was a recipient of IEEE Asia-Pacific Conference on Antennas and Propagation Student Paper Award in 2015, and UK-Europe-China Workshop on Millimeter Waves and $\mathrm{THz}$ Technologies Best Student Paper Award in 2020. He is a reviewer of many journals like IEEE Antennas and Wireless Propagation Letters, IEEE Access, IET Microwaves, Antennas \& Propagation, International Journal of RF and Microwave Computer-Aided Engineering.

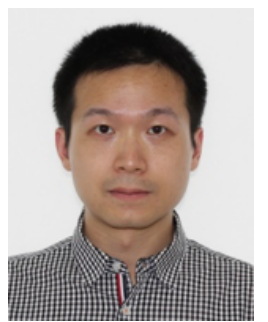

Chao Shu was born in Hefei, Anhui Province, China in 1982. He received the BEng and MEng degrees in communication and information system from Beijing University of Posts and Telecommunication, Beijing, China, in 2005 and 2008, and the Ph.D degree in electronic engineering from Queen Mary University of London in 2021.

From 2008 to 2016, he was with Datang Mobile Communications Equipment Co., Ltd, as a software engineer and then a division manager. His research interests include wideband circularly polarized antennas, millimeter wave and $\mathrm{THz}$ communication and imaging systems. $\mathrm{He}$ has authored a number of papers on these topics, and he holds 11 granted and pending China patents.

Dr Shu is a holder of Project Management Professional (PMP) (2015 present) awarded by the Project Management Institute (PMI). He is a reviewer of several journals like IEEE Antennas and Wireless Propagation Letters, Physical Communication.

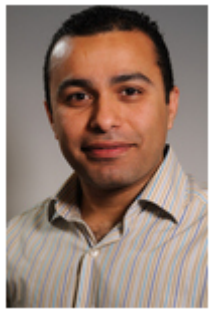

Yasir Alfadhl (M'09-SM'19) was born in Najaf, Iraq, in 1978. He received the B.S. degree and the $\mathrm{Ph} . \mathrm{D}$. degree in telecommunication engineering from the University of London, London, in 2000 and 2006, respecitvely.

Since 2009, he has served as a Lecturer with the School of Electronic Engineering and Computer Science, Queen Mary University of London where he is currently a Senior Lecturer (Associate Professor). He has authored over 100 articles in computational electromagnetics, electromagnetic propagation, high-power microwaves, bioelectromagnetics, and wireless communications. His research activities are focused on antennas and electromagnetic propagations, computational electromagnetics, and bioelectromagnetics.

Dr Alfadhl was a member of the Organizing Committee in the IEEE International Workshop on Antenna Technology: Small and Smart Antennas Conference in Cambridge in 2007. He was nominated (one of the top 20 in the U.K.) by the Institute of Directors for the KTP Business Leader of Tomorrow Awards in 2009.

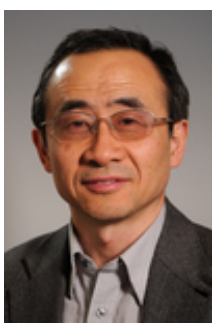

Xiaodong Chen (M'96-SM'07-F'15) was born in Hefei, Anhui Province, China, in 1962. He received the B.Sc. degree in electronics engineering from the University of Zhejiang, Hangzhou, China, in 1983, and the Ph.D. degree in microwave electronics from the University of Electronic Science and Technology of China, Chengdu, China, in 1988

He joined the Department of Electronic Engineering, King's College, University of London, London, U.K., in 1988, as a Postdoctoral Visiting Fellow. In 1990, he was employed by the King's College as a Research Associate and was appointed to a lectureship later on. In 1999, he joined the School of Electronic Engineering and Computer Science, Queen Mary University of London, London, where he is currently a Professor of Microwave Engineering with the School of Electronic Engineering and Computer Science. He is also the Director of the BUPT-QMUL Joint Research Lab, Beijing. He holds a Visiting Professorship with the University of Westminster, U.K., the Beijing University of Posts and Telecommunications (BUPT), and the University of Electronic Science and Technology of China. He has authored or coauthored over 400 publications (book chapters, journal articles, and refereed conference presentations). His current research interests include high-power microwave devices, wireless communications, and antennas.

Dr Chen is a member of the U.K. Engineering and Physical Sciences Research Council Review College and the Technical Panel of the Institution of Engineering and Technology Antennas and Propagation Professional Network. $\mathrm{He}$ is a Fellow of IET/IEEE. 